\title{
Visualization of polarization state and its application in optics classroom teaching
}

Bing Lei, Wei Liu, Jianhua Shi, Wei Wang, Tianfu Yao, et al.

Bing Lei, Wei Liu, Jianhua Shi, Wei Wang, Tianfu Yao, Shugang Liu, "Visualization of polarization state and its application in optics classroom teaching," Proc. SPIE 10452, 14th Conference on Education and Training in Optics and Photonics: ETOP 2017, 1045212 (16 August 2017); doi: 10.1117/12.2269496

SDIE Event: 14th Conference on Education and Training in Optics and Photonics, ETOP 2017, 2017, Hangzhou, China 


\title{
Visualization of polarization state and its application in Optics classroom teaching
}

\author{
Bing Lei ${ }^{1, *}$, Wei Liu ${ }^{1}$, Jianhua Shi ${ }^{1}$, Wei Wang ${ }^{1}$, Tianfu Yao ${ }^{1}$ and Shugang Liu ${ }^{2}$ \\ ${ }^{1}$ College of Optoelectronic Science and Engineering, National University of Defense Technology, \\ Changsha, Hunan 410073, China \\ ${ }^{2}$ Heilongjiang University, Harbin, Heilongjiang 150080, China
}

\begin{abstract}
Polarization of light and the related knowledge are key and difficult points in optical teaching, and they are difficult to be understood since they are very abstract concepts. To help students understand the polarization properties of light, some classroom demonstration experiments have been constructed by employing the optical source, polarizers, wave plates optical cage system and polarization axis finder (PAF). The PAF is a polarization indicating device with many linear polarizing components concentric circles, which can visualize the polarization axis's direction of linearly polarized light intuitively. With the help of these demonstration experiment systems, the conversion and difference between the linear polarized light and circularly polarized light have been observed directly by inserting or removing a quarter-wave plate. The rotation phenomenon of linearly polarized light's polarization axis when it propagates through an optical active medium has been observed and studied in experiment, and the strain distribution of some mounted and unmounted lenses have also been demonstrated and observed in experiment conveniently. Furthermore, some typical polarization targets, such as liquid crystal display (LCD), polarized dark glass and skylight, have been observed based on PAF, which is quite suitable to help students understand these targets' polarization properties and the related physical laws. Finally, these demonstration experimental systems have been employed in classroom teaching of our university in physical optics, optoelectronics and photoelectric detection courses, and they are very popular with teachers and students.
\end{abstract}

Keywords: Visualization of polarization state, classroom demonstration experiment, polarization axis finder, lens strain detection, optics classroom teaching

\section{INTRODUCTION}

As we all known, light waves traveling in free space or another homogeneous isotropic non-attenuating medium, are properly described as transverse waves, and polarization is the basic performance of electromagnetic wave. However, polarization of light is important and difficult points in optical classroom teaching, and the related knowledge, such as linearly polarized light, circularly polarized light, elliptically polarized light, degree of polarization, polarization angle and direction, are all difficult to be understood since they are non-intuitive concept and really difficult to be observed in experiment [1]. To help students understand the polarization properties of light, a number of classroom demonstration experiments have been constructed by utilizing the optical source, polarizers, wave plates, polarization axis finder (PAF) and optical cage system. The PAF is an unique tool that provides instant indication of the laser beam's polarization axis, and not only no twisting or turning is required, but also measuring minimum and maximum power levels with a power meter is also not needed. The actual direction of the electric vector (i.e. axis of polarization) is indicated directly by an "hourglass" or "bowtie" pattern in the beam [2].

In this paper, some polarization related classroom teaching demonstration experimental systems have been constructed to help students understand the key and difficult knowledge points of polarization based on PAF. The working principle and main features of PAF has been introduced firstly. Two different types of polarization axis visualization systems have been made to observe the polarization direction of a light beam, and the optical activity and strain detection experiments have been demonstrated based on the experimental configuration. Finally, the polarization properties' of LCD, polarized dark glass and skylight have also been studied by PAF.

*leibing_2000@126.com; phone 086 0731-84574749

14th Conference on Education and Training in Optics and Photonics: ETOP 2017, edited by Xu Liu,

Xi-Cheng Zhang, Proc. of SPIE Vol. 10452, 1045212 - @ 2017 ICO, IEEE, OSA, SPIE

CCC code: $0277-786 X / 17 / \$ 18 \cdot$ doi: $10.1117 / 12.2269496$ 


\section{VISUALIZATION OF POLARIZATION STATE}

The polarization axis finder (PAF) is a unique tool with many linear polarizing components concentric circles [3], and real object and schematic diagram are shown in Fig. 1(a) and (b). PAF can be used conveniently, which can indicate the polarization axis' direction of linearly polarized light intuitively and instantly, and the changes of polarization state and its stability can also be monitored directly. Moreover, no twisting or rotating is required, and no power meter or photo detector is needed since there is no need to measure minimum and maximum power levels.

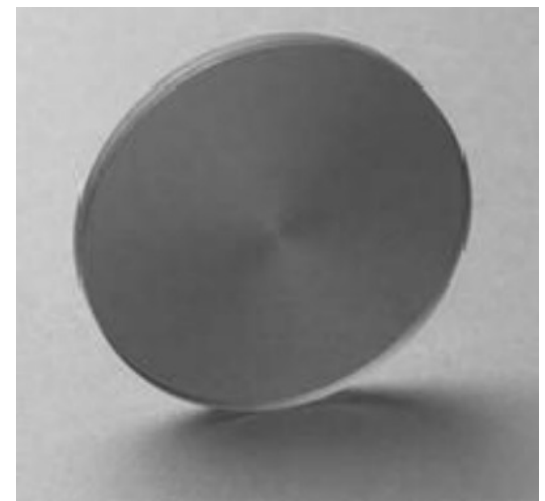

(a)

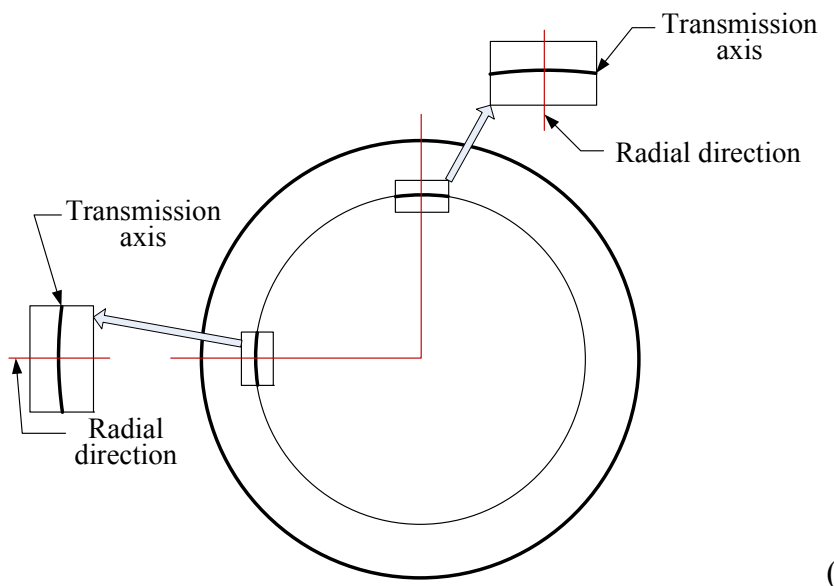

(b)

Fig. 1 (a) the real object and (b) schematic diagram polarization axis finder (PAF).

The working principle of a polarization axis finder indicating the light beam's polarization direction is shown in Fig. 2. An optical source emits unpolarized light without specific polarization axis, and a polarizer is utilized to transfer the unpolarized light into linearly polarized light, then the linearly polarized light's polarization axis, i.e. the actual direction of the electric vector is indicated directly by an "hourglass" or "bowtie" pattern in the beam by PAF. Since the PAF is consist of many linear polarizing components concentric circles, and the direction of these tiny linearly polarizers are perpendicular to the radial direction, therefore it is also called angular polarizer. If the small component linearly polarizer is parallel to the direction of input linearly polarized light's axis, in other words, the radial direction is perpendicular to the input beam's polarized axis, the light beam can pass PAF completely and form the bright part of the above "bowtie" pattern according to the Malus law. Oppositely, when the small component linearly polarizer is perpendicular to the direction of input linearly polarized light's axis, the light beam will be completely blocked by the PAF, and the dark area will be formed in the pattern. The rest polarizers with polarization axis in other directions will partially transmit the input light beam, and form the gradual gray level part of the above "bowtie" pattern.

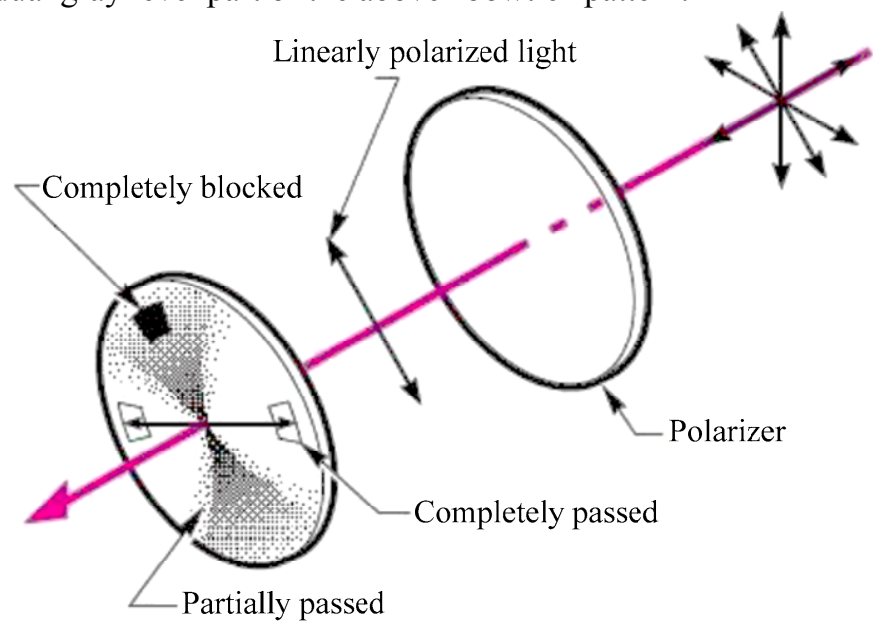

Fig. 2 The PAF's working principle of indicating the polarization axis of linearly polarized light. 
The experimental configuration of the polarization demonstration system is shown in Fig. 3, and the real object and its effect on polarization indication are shown in Fig. 4(a) and (b) respectively. The demonstration experimental setup is portable and easy to use, and it is quite suitable for classroom teaching and demonstration experiments. Based on the demonstration experimental setup, a series of polarization related phenomena have been observed conveniently. Firstly, the polarization axis's direction of linear polarized light has been observed intuitively. Secondly, the linear polarized light has been conversed to circularly polarized light by inserting a quarter-wave plate, and well-distributed pattern in the beam has been obtained in experiment, which means that no fixed polarization axis exist in circularly polarized light. It is helpful for students to understand the difference between linearly polarized light and circularly polarized light. Thirdly, the strain distribution of a lens has also been demonstrated and observed in experiment conveniently.

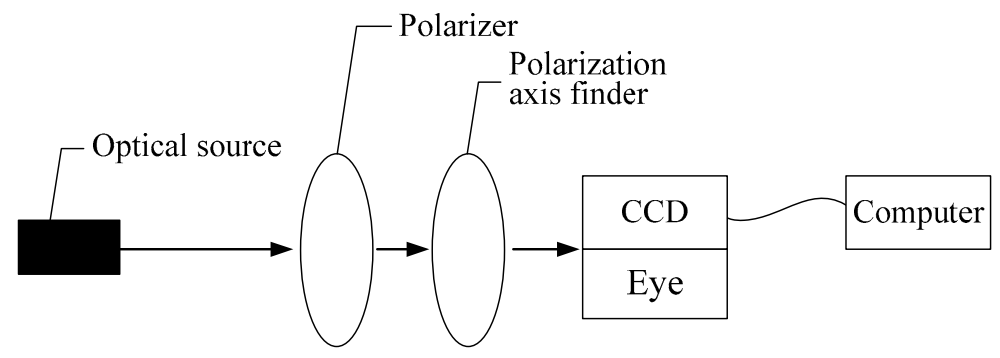

Fig. 3 Experimental configuration of the polarization demonstration system.
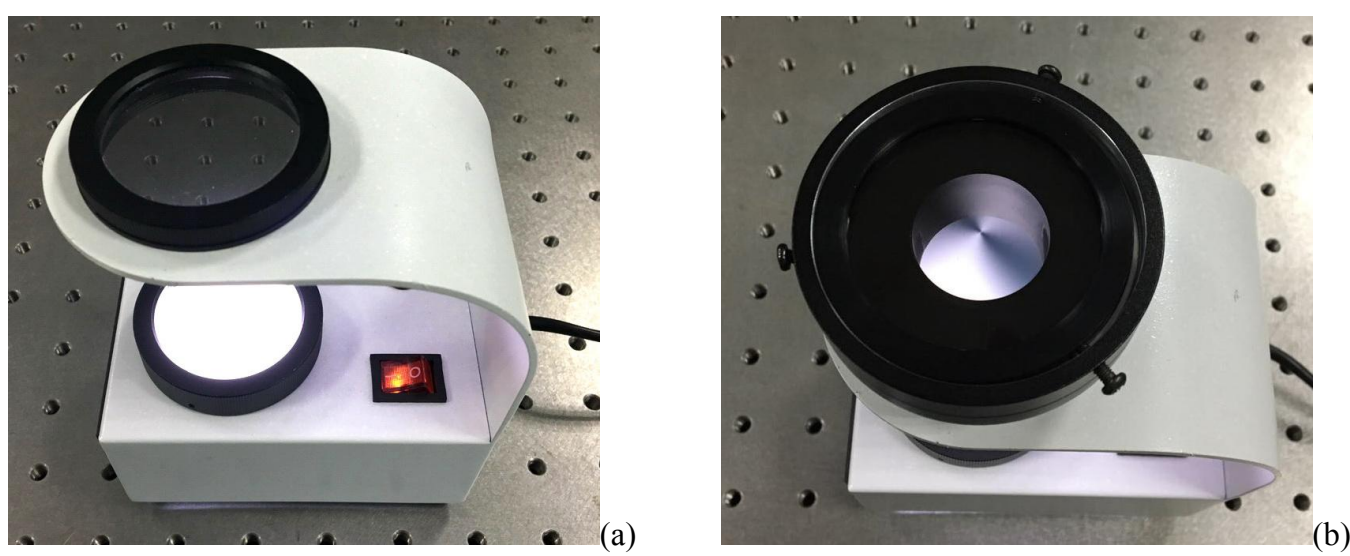

Fig. 4 (a) the real object of experimental configuration and (b) its effect of polarization indication.
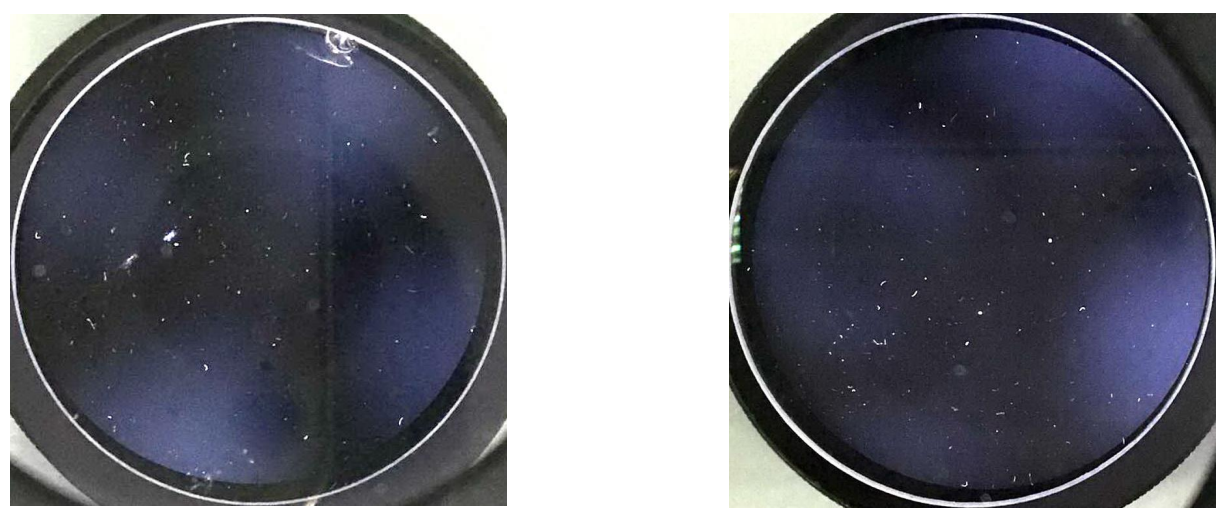

Fig. 5 Two unmounted lens with different strain distribution.

The examining configuration of lens strain distribution has been obtained by simply improving the above system. Two polarizers with orthogonal polarization axis have been placed in a certain distance, and the lens needed to be checked is 
set between the two polarizers. If there is uneven strain distribution in the lens, the extra phase difference between two orthogonal polarized modes will be introduced, and uneven intensity distribution will be observed. Two unmounted lens have been examined with the configuration, and the experimental results are shown in Fig. 5. A mounted lens and glasses have also been checked by this setup, and the results are shown in Fig. 6. The red circle shown in Fig. 6 includes a fixed screw, which induces great strain in the lens, and the strain can be indicated obviously in our experimental setup. Based on the experiments, we have clearly found that obvious and large strain exists in the mounted lens due to the mounting pressure, and it provides a simple and effective method to check the mounting quality of a lens.
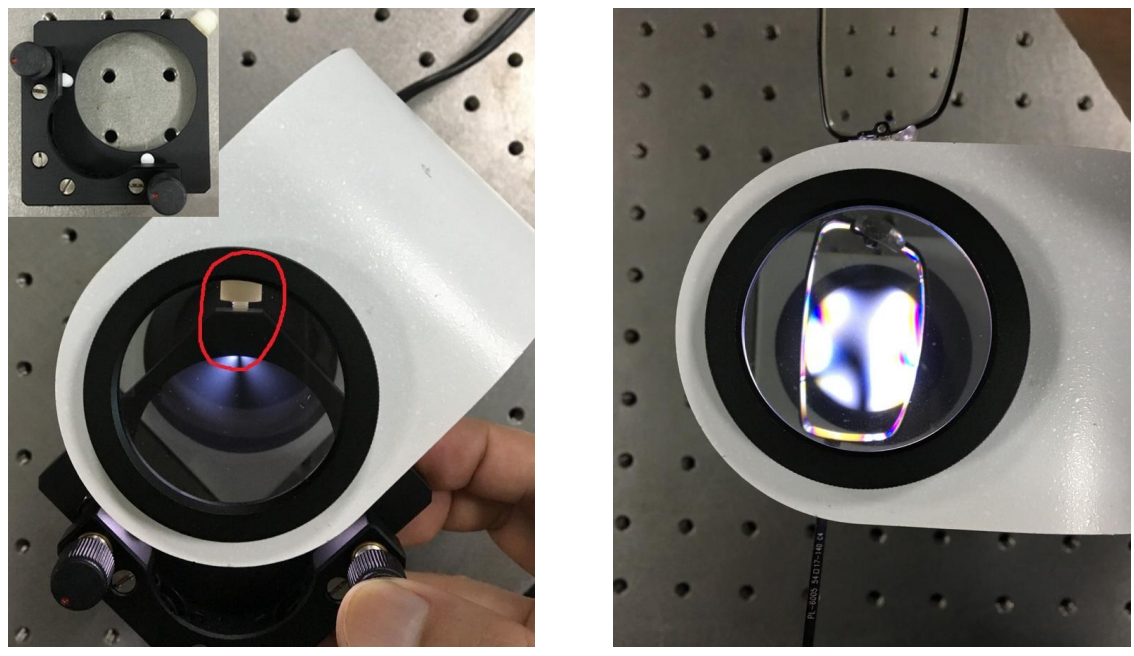

Fig. 6 A mounted lens and glasses with obvious and large strain distribution.

Another version of the experimental configuration which can be utilized to investigate the medium's optical activity and indicate the beam's polarization direction has been constructed based on the optical cage system. As we all known, the glucose solution is a typical optical active medium, which is employed as samples to study the optical activity law by using our experimental system, and the experimental setup and results are shown in Fig. 7. According the demonstration experiment, we can reveal the relationship between optical active degree and optical length of glucose solution clearly, and it is quite convenient for students to understand the optical activity law. On the other hand, the solubility of glucose solution can be obtained by measuring the rotation angle of polarization axis precisely, and the measurement of rotation angle can be done by various digital image processing methods.

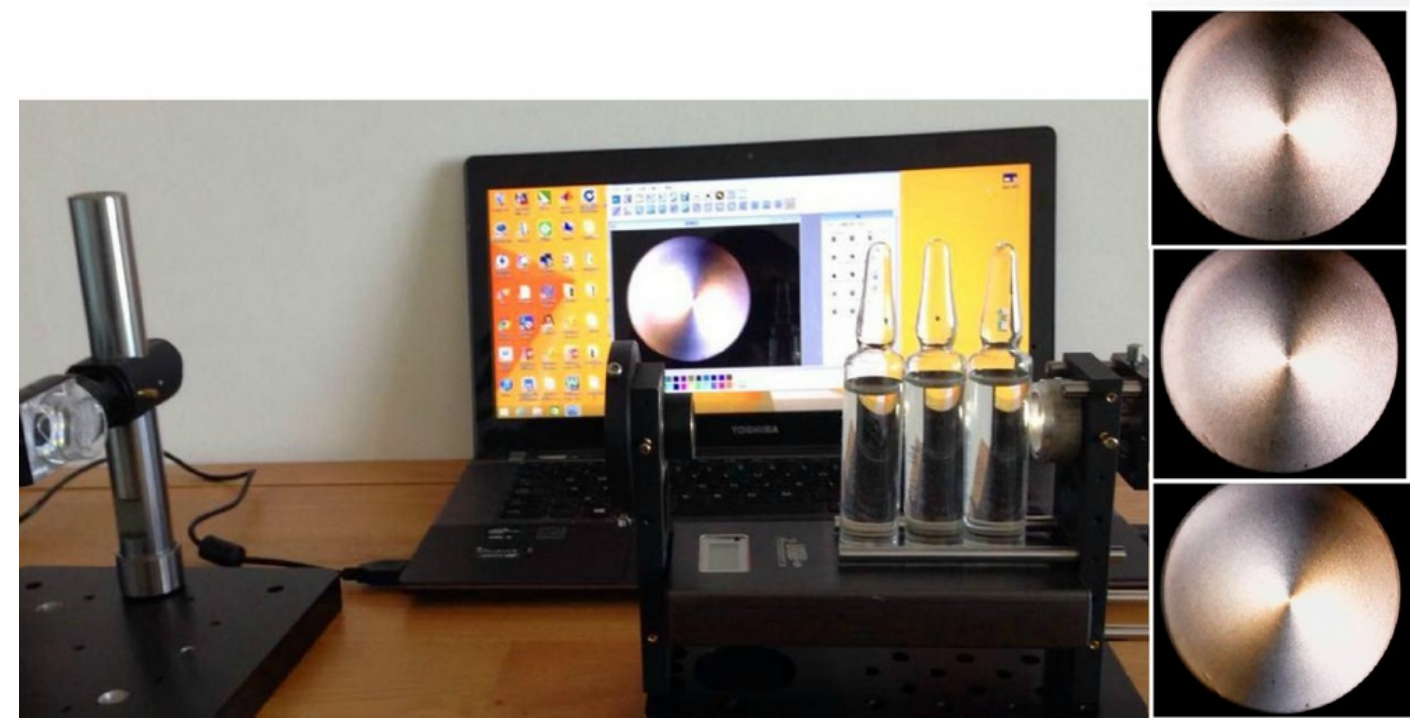

Fig. 7 A mounted lens and glasses with obvious and large strain distribution. 


\section{THREE INTERESTING EXAMPLES ABOUT POLARIZATION}

Based on the polarization axis finder (PAF), three intuitive and interesting examples about polarization detection and its applications have been introduced and discussed, which are easy to implement and are welcome by teachers and students, and they are very suitable for exciting the students' study interest and understanding the physical law of polarization.

The first example is the polarized dark glass. The polarized dark glass can be regarded as a polarizer, and thus whether the dark glass is real polarized or not can be checked by the PAF. Based on the example, the Brewster's law and the polarization state of reflection light can be instructed easily, and some thinking questions can be proposed for further discussion, such as the polarized dark glass's axis should be set in horizontal direction or in vertical direction, why the polarized dark glass can make the user feel comfortable and which conditions is quite necessary to wear the polarized dark glass. Fig. 8 is the checking result of the polarized dark glass with PAF.

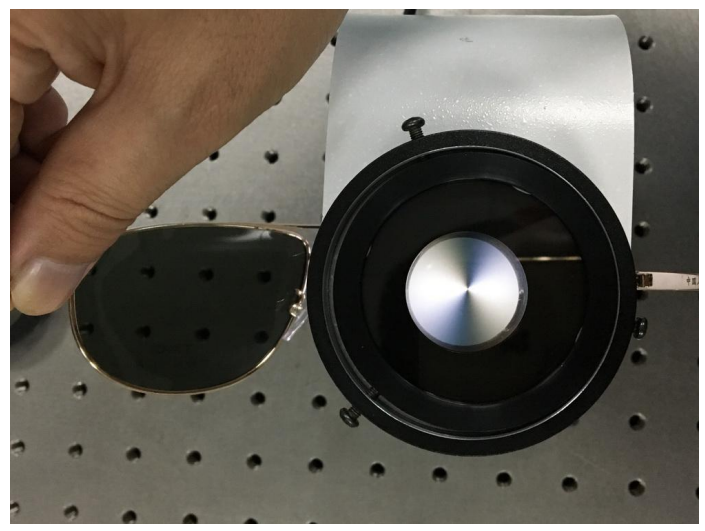

Fig. 8 The checking results of the polarized dark glass with PAF.

The second example is the liquid crystal display (LCD). Some students may not know the emitting light of LCD is linearly polarized light, but the truth can be examined by PAF conveniently. This example can not only help students understand the structure and working principle of LCD, but also make them confirm the LCD's polarization properties. Fig. 9(a) and (b) are the observed results of two different LCDs with PAF.
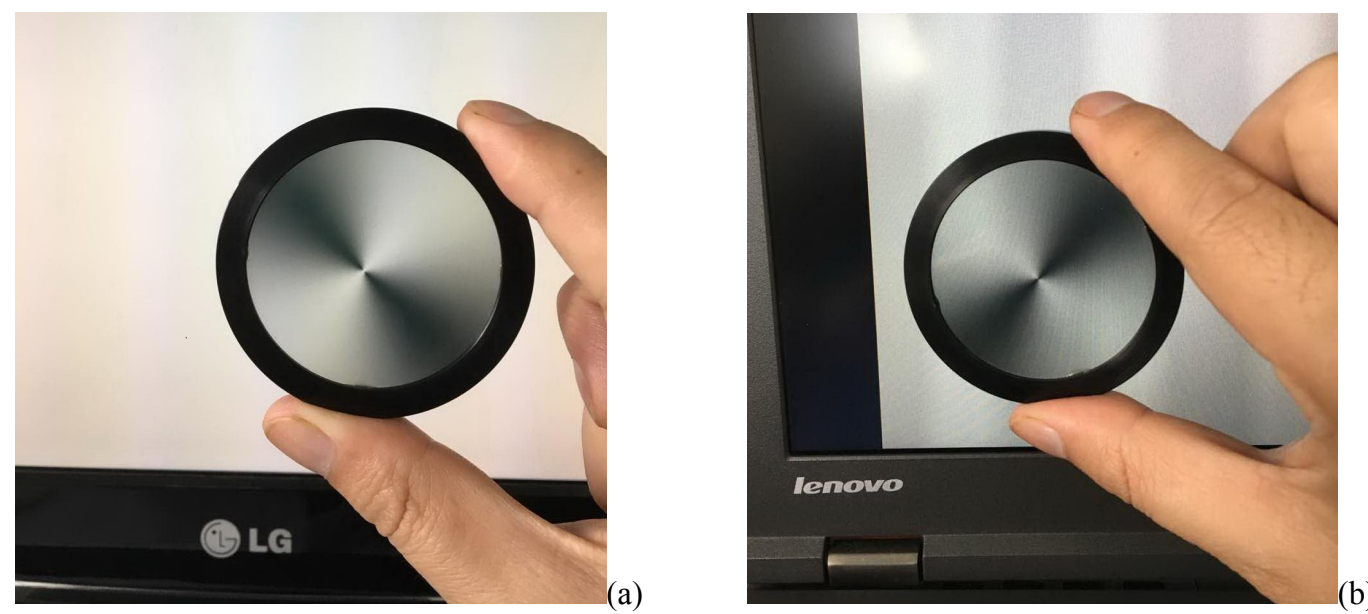

Fig. 9 The observed results of two different LCD displays with PAF.

The third example is the sky polarization mode. The natural light of sky shows partial degree of polarization due to the Rayleigh scattering, especially in a sunny day, which can also be observed by the PAF, and the experimental results is shown in Fig.10. According to the observed results, we can clearly find that the sky scatting light owns regular law in polarization direction, and investigating the sky polarizing law helps engender some important application fields, such as the polarized skylight navigation [4-5]. 


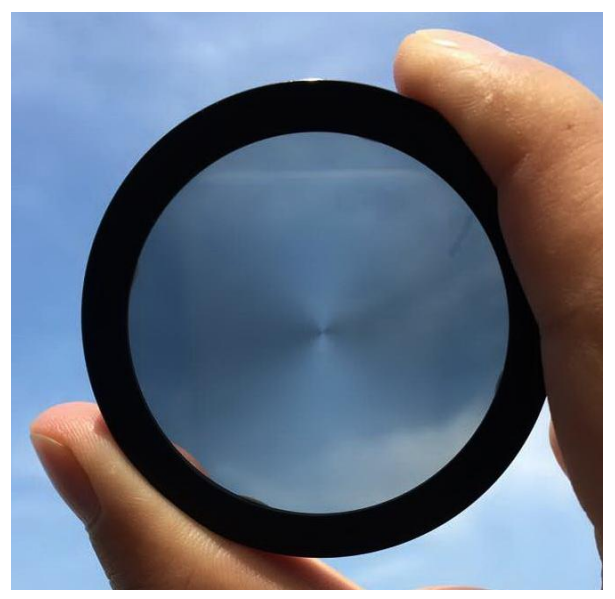

Fig. 10 The sky polarization mode observed by PAF.

\section{CONCLUSION}

The classroom demonstration experiment is not only an effective way to grasp students' attention and excite their study interest, but also a good method to help students understand the difficult and key knowledge points correctly. Some polarization related classroom teaching demonstration experimental systems have been constructed based on a unique and useful tool PAF which can provide instant indication of the laser beam's polarization axis. Two different types of polarization axis visualization systems have been made to observe the polarization direction of a light beam. The optical activity of glucose solution has been studied based on the demonstration experimental system, and the strain distribution of some mounted and unmounted lenses have also been demonstrated and observed in experiment. Finally, three intuitive and interesting examples, LCD, polarized dark glass and skylight, have been observed and discussed based on PAF, which is quite suitable to help students understand these targets' polarization properties and the related physical laws.

\section{Acknowledgments}

This work is supported by the education and teaching research undergraduate project of National University of Defense Technology (Grant No. U2016007) and the education and teaching reform research project of College of Optoelectronic Science and Engineering, National University of Defense Technology (Grant No. 2015XY03).

\section{REFERENCES}

[1] Q. D. Yuan, C. Y. Li, “A portable demonstrator of polarized light in class," Journal of Hangzhou Normal University (Natural Science Edition) 14(4), 446-448 (2015).

[2] http://www.tsi.com/Products/_Product-Accessories/Polarization-Axis-Finder-10901A.aspx.

[3] Y. Huang, H. A. Ye, L. X. Gao, M. Zhang, S. G. Liu, "New method of generating vectorial polarized beams," Chinese Journal of Lasers 39(4), 17-20 (2012).

[4] W. J. Zhang, Y. Cao, X. Z. Zhang, Z. J. Liu, "Sky light polarization detection with linear polarizer triplet in light field camera inspired by insect vision," Applied Optics, 54(30), 8962-8970(2015).

[5] Y. J. Wang, X. P. Hu, J. X. Lian, L. L. Zhang, X. F. He, "Mechanisms of bionic positioning and orientation based on polarization vision and corresponding experiments," Optics and Precision Engineering, 24(9), 2109-2116(2016). 